\title{
Projection of ST segment changes on to the front of the chest \\ Practical implications for exercise testing and ambulatory monitoring
}

\author{
K M FOX, J DEANFIELD, P RIBERO, D ENGLAND, C WRIGHT \\ From Division of Cardiovascular Disease, Hammersmith Hospital, DuCane Road, London
}

SUMMARY In order to determine the optimal chest leads for exercise testing and ambulatory monitoring we have studied the projection of ST segment alterations on to the front of the chest in 200 patients. In 20 of these patients recordings during exercise were analysed to study the pattern of changes of the ST segment during and after exercise. The electrocardiogram was recorded using 16 unipolar chest leads equally spaced over the left hemithorax. ST segment elevation and depression projected to the front of the chest in a pattern that was individual to each patient and indeed the standard chest leads of the 12 lead electrocardiogram were seen to represent only $41 \%$ of the total projection. ST segment depression was usually first recorded in one or two leads and the number of leads involved increased as exercise progressed, returning to normal after exercise in a reciprocal fashion; the leads that first showed ST segment depression were almost invariably those with the most severe depression and the last in which it disappeared. Analysis of the precordial projection of the ST segment has allowed a 12 lead precordial matrix to be designed that can be recorded using a standard three channel electrocardiographic machine. This will provide important directional information about the severity of myocardial ischaemia when assessing the effects of interventions that would not otherwise be available using conventional electrocardiographic lead systems. It is also clear from these data that failure to record ST segment changes using ambulatory monitoring in patients complaining of chest pain may mean that the wrong site was selected for placing the exploring electrode. Moving one or both of the exploring electrodes to other positions of the precordial matrix in subsequent recordings may then provide diagnostic information that otherwise would have been missed.

In recent years there has been increasing interest in the use of non-invasive tests to diagnose and evaluate patients with ischaemic heart disease. Exercise testing has proved to be the most useful and widely available technique and more recently 24 hour ambulatory monitoring of ST segments has provided a means of evaluating patients in their everyday life. ${ }^{1-3}$ Initially only a single chest lead was used for exercise testing and many limitations of the technique were resolved by using the standard 12 lead electrocardiogram. ${ }^{4}$ More recently we and others have found it more useful to use a 16 lead precordial mapping system for both the diagnosis and the evaluation of patients with ischaemic heart disease. ${ }^{5-7}$ In spite of a vast amount of research on the value of exercise testing in identifying coronary artery disease there has been little work describing the pattern of ST segment projection on to the front of the chest during an exercise test. Without this information many of the strengths and weaknesses of exercise testing and ambulatory monitoring will remain speculative.

\section{Methods}

\section{PATIENTS}

Two hundred consecutive patients (170 men, 30 women) undergoing diagnostic coronary angiography were studied. One hundred and eighty two patients presented with chest pain. There were 18 patients without chest pain in whom the angiograms were performed for diagnostic purposes. They were aged 29 to 69 (mean $48 \cdot 2 \pm 8 \cdot 6$ ) years. 
PRECORDIAL ELECTROCARDIOGRAPHIC MAPPING All patients underwent 16 lead precordial electrocardiographic mapping before, immediately after exercise, and 10 minutes after exercise using a technique previously described. ${ }^{5}$ The 16 points were distributed evenly over the left hemithorax and the boundaries were the angle of Louis, the right sternoclavicular joint, the posterior axillary line, and $6 \mathrm{~cm}$ below the xiphisternum (Fig. 1). The leads were unipolar chest leads connected via a Wilson central terminal. Graded maximal exercise tests were performed using a bicycle ergometer. Patients cycled at a constant speed (50 revolutions per minute) and the work load was increased by 25 watts each minute. The exercise tests were limited by the appearance of chest pain, dyspnoea, fatigue, and multiple ventricular ectopic complexes.

In 20 of the patients with proven coronary disease and ST segment depression, 16 lead precordial maps were recorded each minute during exercise.

\section{INTERPRETATION OF RECORDS}

At each of the 16 positions ST segment depression and elevation were measured in three complexes and an average taken. ST segment depression was measured in millimetres (mm) to the nearest $0.5 \mathrm{~mm}$. The ST segment was considered depressed if there was a change of $1 \mathrm{~mm}$ or more lasting $0.08 \mathrm{~s}$ or longer. The ST segment was considered elevated if there was a change of $1 \mathrm{~mm}$ or more measured $0.06 \mathrm{~s}$ after the end of the QRS complex.

One of the precordial positions (C3) corresponded exactly to V5 and five of the 16 precordial leads (A2, B2, B3, C3, D3) covered the positions usually occupied by the chest leads of the standard 12 lead electrocardiogram (modified standard leads) ${ }^{8}$ All data from the exercise tests were reported before the coronary arteriograms were obtained.

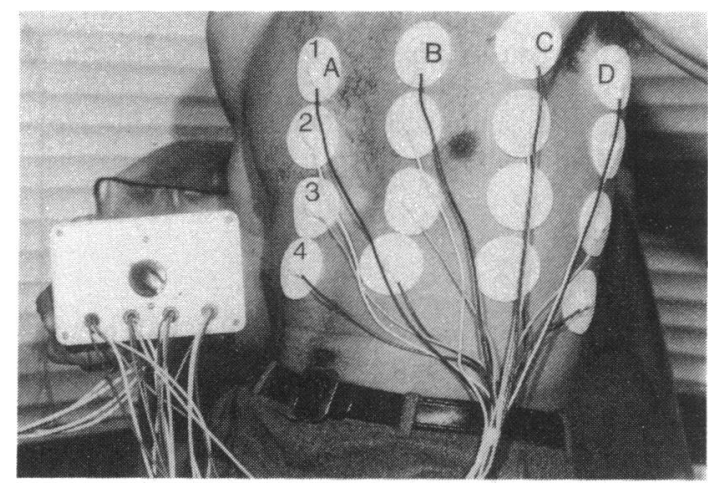

Fig. 1 The electrodes are distributed over the left hemithorax and labelled $A$ to $D$ (vertical rows) and 1 to 4 (horizontal rows).

\section{CORONARY ARTERIOGRAPHY}

All patients had left ventricular angiograms and coronary arteriograms performed using the Judkins technique. Coronary arterial narrowing was considered significant where the luminal diameter was decreased by at least $70 \%$.

\section{Results}

Satisfactory recordings were obtained in all patients. The results of exercise testing and coronary arteriography are shown in Table 1. ST segment depression was found to occur in 122 of the 154 patients with coronary disease. A further 12 patients developed ST segment elevation alone.

Projection of ST segment depression to the 16 precordial leads is shown in Table 2. ST segment depression following exercise was projected to all 16 leads. ST segment depression was frequently recorded outside both V5 and the modified standard chest leads. Indeed ST segment depression recorded in the standard precordial leads represented only $41 \%$ of the total projection to the full 16 leads.

ST segment depression occurred exclusively outside the conventional precordial leads in nine of the 122 (7\%) patients who developed ST segment depression and had coronary disease. Maximum ST segment depression occurred outside the conventional leads in $38(31 \%)$ patients. In $21(17 \%)$ patients ST segment depression was recorded outside the two leads (V5 and inferior) used for ambulatory monitoring.

Projection of ST segment elevation to the 16 precordia leads is shown in Table 3. Again ST segment elevation

\section{Table 1 Clinical details}

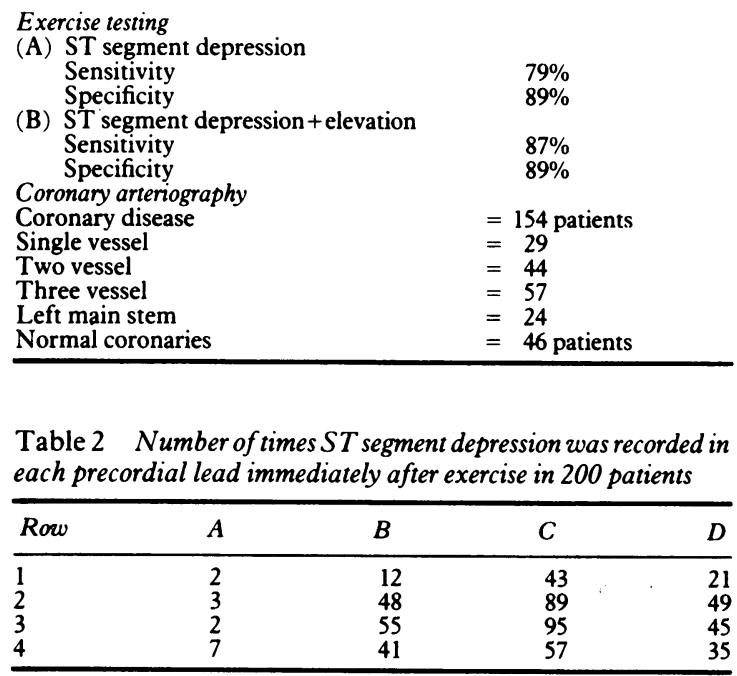


after exercise was often recorded in leads other than those usually occupied by the standard chest leads (that is ST segment elevation recorded in the standard precordial leads represented only $42 \%$ of the total projection to the 16 precordial leads).

False positive exercise-induced ST segment depression was recorded in almost all of the 16 precordial leads and was not confined or more frequent in any particular set of leads (Table 4). ST segment elevation in patients without prior myocardial infarction was confined to the right chest leads and was found in eight patients; six of these patients had ST segment depression also (Table 5). All patients with exercise-induced ST segment elevation had coronary disease at coronary angiography.

\section{PATTERN OF PROJECTION OF ST SEGMENT CHANGES}

In all 20 patients in whom recordings were made during exercise ST segment depression was first recorded in a small number of leads and increased in area as exercise continued. After the end of exercise, after an initial further increase in the number of leads with ST segment depression in eight $(40 \%)$ patients, the area decreased. The first lead with ST segment depression was invariably the lead with the most severe ST segment depression at peak exercise and the same lead

Table 3 Number of times ST segment elevation was recorded in each precordial lead immediately after exercise in 200 patients

\begin{tabular}{lcccc}
\hline Row & $A$ & $B$ & $C$ & $D$ \\
\hline 1 & 18 & 17 & 11 & 1 \\
2 & 22 & 20 & 13 & 1 \\
3 & 11 & 15 & 8 & 1 \\
4 & 3 & 7 & 5 & 0 \\
\hline
\end{tabular}

Table 4 Number of times false positive ST segment depression was recorded in each precordial lead immediately after exercise in four patients

\begin{tabular}{lllll}
\hline Row & $A$ & $B$ & $C$ & $D$ \\
\hline 1 & 0 & 1 & 0 & 0 \\
2 & 0 & 1 & 2 & 2 \\
3 & 0 & 3 & 3 & 1 \\
4 & 1 & 3 & 3 & 1 \\
\hline
\end{tabular}

Table 5 Number of times $S T$ segment elevation was recorded in each precordial lead immediately after exercise in eight patients without previous myocardial infarction

\begin{tabular}{lllll}
\hline Row & $A$ & $B$ & $C$ & $D$ \\
\hline 1 & 8 & 4 & 0 & 0 \\
2 & 8 & 3 & 0 & 0 \\
3 & 4 & 0 & 0 & 0 \\
4 & 1 & 0 & 0 & 0 \\
\hline
\end{tabular}

Table 6 Number of times $S T$ segment changes zere confined to individual vertical or horizontal rows

\begin{tabular}{llll}
\hline Row & $\begin{array}{l}\text { ST segment } \\
\text { depression }\end{array}$ & $\begin{array}{l}\text { ST segment elevation } \\
\text { with past myocardial } \\
\text { infarction }\end{array}$ & $\begin{array}{l}\text { ST segment elevation } \\
\text { without past myocardial } \\
\text { infarction }\end{array}$ \\
\hline A & 0 & 0 & 2 \\
B & 6 & 1 & 0 \\
C & 20 & 0 & 0 \\
D & 1 & 0 & 0 \\
1 & 3 & 0 & 1 \\
2 & 1 & 0 & 0 \\
3 & 2 & 2 & 0 \\
4 & 4 & 0 & 0 \\
\hline
\end{tabular}

was the last to return to normal. An example is shown in Fig. 2.

Analysis of the ST segment projection immediately after the end of exercise in 200 patients showed that on only five occasions was the ST segment alteration confined to one lead (those were C2, C3, C4, B3, B4). The number of times ST segment alterations were confined to one of the horizontal or vertical rows is shown in Table 6. Though ST segment changes in vertical row $A$ occurred 29 times, on no occasion was ST segment depression confined to this row. In two patients ST segment elevation was seen only in row A.

\section{Discussion}

In 1932, Wolferth and Wood ${ }^{9}$ described the first chest lead that proved of value in clinical medicine and their work stimulated clinical interest in precordial leads. They obtained a recording which they called the fourth lead (lead IV) by placing the left arm electrode over the apex of the heart and connecting the galvanometer to the lead III circuit. After this many other new leads were introduced including those based on the concept of the "exploring" electrode and "indifferent" electrode, as described by Wilson and his associates. ${ }^{10}$ Many different areas were suggested for the exploring electrode, but the majority were over the anterior surface of the chest, particularly over the area of the heart. By convention the exploring electrode was arbitrarily placed in the standard six positions now used, connected through a central terminal. ${ }^{10}$ Thus, there followed the CT 1-6 series of leads which, for convenience, were labelled V1-6 as presently used. In almost 50 years which have followed there has been no study investigating the relative merits of these standard lead positions in identifying ST segment abnormalities.

In recent years there has been a surge of interest in non-invasive diagnosis in cardiology, and, in particular, exercise testing and ambulatory monitoring are being used with increasing frequency. 1112 If we are to make optimal use of these techniques, however, it is necessary to know much more about the projection of 


\section{During Exercise}

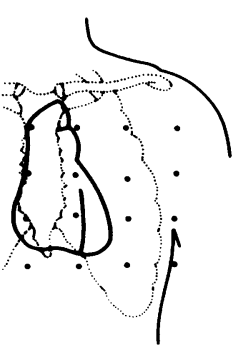

Control

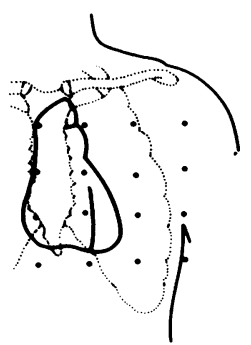

1 minute

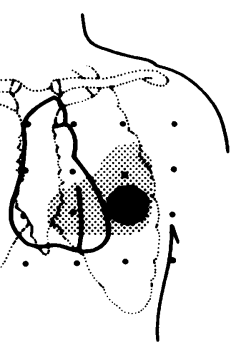

2 minutes

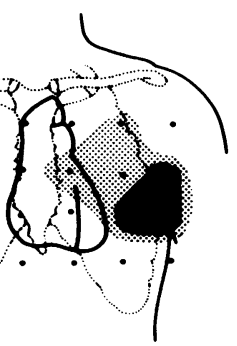

3 minutes

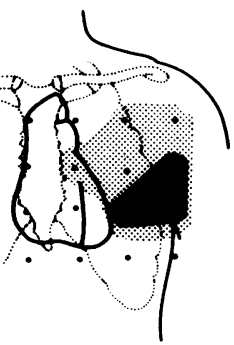

Peak Exercise

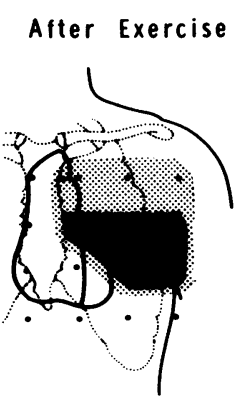

Immediately

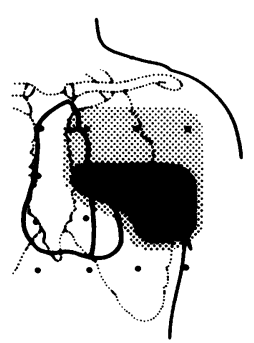

1 minute

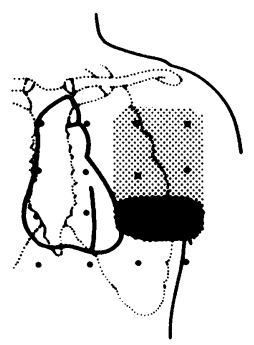

3 minutes

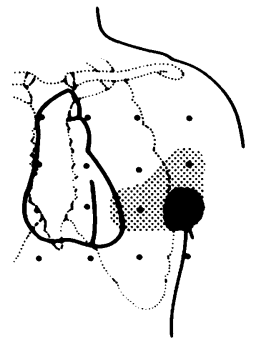

5 minutes

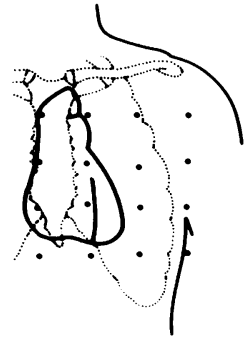

10 minutes

$$
1 \mathrm{~mm} \geqslant 2 \mathrm{~mm}
$$

Fig. 2 A sequence of contour maps has been drawn of the projection of ST segment depression on to the front of the chest in a single patient. The first leads in which ST segment depression was recorded were those with the most severe $S T$ segment depression at peak exercise and the last to return to normal after exercise.

ST segment abnormalities on to the front of the chest. Whether only a single lead or multiple leads are used for the exercise test, it is necessary to have some concept of the pattern and distribution of ST segment changes in patients with ischaemic heart disease; the same holds true particularly in ambulatory monitoring where there are only two exploring electrodes available.

We have studied the projection of ST segment changes during and after a maximal exercise test using 16 precordial leads arranged over the left hemithorax in a matrix. The ST segment projects on to the front of the chest during exercise in a uniform manner. The area (that is number of leads involved) increases as exercise progresses and then shrinks after exercise in a reciprocal fashion. In a substantial number of patients the area continues to increase for up to three minutes after exercise before decreasing. The lead that first shows the ST segment depression is almost invariably the lead in which the most severe ST segment depression is found and the last in which it disappears. This suggests that ischaemia starts in one site, increases in area as the provocation continues, and then afterwards gradually recedes. The use of multiple leads arranged in a matrix will therefore have advantages in determining the effects of interventions over the conventional, arbitrarily placed chest leads.

This study has shown that ST segment depression in an exercise test will project over the front of the chest in a pattern that is individual to each patient. The projection will be related to the site, severity, and extent of ischaemia in each patient and be modified by a number of non-cardiac factors, that is chest size and shape, resistance of tissue between the heart and chest wall, etc. Projection of ST segment depression involving the standard leads of the electrocardiogram was seen to represent only $41 \%$ of the total projection; and ST segment elevation to represent $42 \%$. In $31 \%$ maximal ST segment alteration was projected outside the area subtended by the standard leads and in $19 \%$ there were no ST segment alterations in the two standard positions usually selected for $\mathbf{2 4}$ hour monitoring.

Ambulatory monitoring of ST segments is performed using recorders that have two exploring electrodes; usually these are placed in an anterior (V5) and inferior site. Failure to record ST segment changes in patients complaining of chest pain may thus mean that the wrong site has been selected for placing the exploring electrode. Previous studies have shown that 
to obtain the maximal information from ST segment ambulatory monitoring it is necessary to record not only from a site where ST segment depression is known to occur but where it occurs maximally. Moving one or both of the exploring electrodes to a more superior position of our 16 lead matrix in subsequent recordings may then provide diagnostic information that otherwise would have been missed.

Almost all electrocardiograph machines used for exercise testing have three recording channels. In this study we have analysed the precordial projection to determine if a matrix arrangement can be maintained in such a way that the recording could be performed with a three channel electrocardiogram machine without loss of important data. Row A was involved in $35 \%$ of the total projection of ST segment elevation and $2 \%$ of the total projection of ST segment depression. In only two patients were the ST segment alterations confined to this row and in none was ST segment depression seen only in this row. We, therefore, recommend that a precordial mapping system could be satisfactorily obtained using 12 precordial leads (rows B, C, and D). Recording of the limb leads should also be performed' since, though inferior changes are usually projected to the bottom row of the map, recording the inferior limb leads would ensure no information is lost.

In 1967 Mason and his colleagues ${ }^{11}$ introduced the standard 12 leads into exercise testing. We now recommend that an 18 lead system using 12 precordial leads arranged as a matrix has important advantages, particularly as it allows the precordial projection of ST segment changes to be evaluated in each individual patient. This may be undertaken without requiring any new equipment other than a switching box and a little extra effort in placing the electrodes.

\section{References}

1 Rosing DR, Reichek N, Perloff JK. The exercise test as a diagnostic and therapeutic aid. Am Heart $\mathcal{F} 1974 ; 87$ : 584-96.

2 Schang ST Jr, Pepine CJ. Transient asymptomatic S-T segment depression during daily activity. Am $\mathcal{f}$ Cardiol 1977; 39: 396-402.

3 Selwyn AP, Fox KM, Eves M, Oakley D, Dargie H, Shillingford JP. Myocardial ischaemia in patients with frequent angina pectoris. $\mathrm{Br}$ Med F 1978; ii: 1594-6.

4 Mason RE, Likar I. A new system of multiple-lead exercise electrocardiography. Am Heart f 1966; 71: 196205.

5 Fox KM, Selwyn AP, Shillingford JP. Precordial exercise mapping: improved diagnosis of coronary artery disease. Br Med F 1978; ii: 1596-8.

6 Lynch P, Dargie H, Krikler S, Krikler D. Objective assessment of antianginal treatment: a double blind comparison of propranolol, nifedipine and their combination. Br Med F 1980; 281: 184-7.

7 Dawson JR, Whitaker NHG, Sutton GC. Calcium antagonist drugs in chronic stable angina. Comparison of verapamil and nifedipine. Br Heart $\mathcal{F} 1981$; 46: 508-12.

8 Fox KM, Selwyn A, Oakley D, Shillingford JP. Relationship between the precordial projection of S-T segment changes after exercise and coronary angiographic findings. Am $\mathcal{F}$ Cardiol 1979; 44: 1068-75.

9 Wolferth CC, Wood FC. The electrocardiographic diagnosis of coronary occlusion by the use of chest leads. Am 7 Med Sci 1932; 183: 30-5.

10 Wilson FN, Johnston FD, MacCleod AG, Barker PS. Electrocardiograms that represent the potential variations of a single electrode. Am Heart f 1934; 9: 447-58.

11 Mason RE, Likar I, Biern RO, Ross RS. Multiple lead exercise electrocardiography. Circulation 1967; 36: 51725.

12 Balasubramanian V, Lahiri A, Green HL, Stott FD, Raftery EB. Ambulatory S-T segment monitoring. Problems, pitfalls, solutions and clinical application. $\mathrm{Br}$ Heart F 1980; 44: 419-25.

Requests for reprints to Dr K Fox, National Heart Hospital, Westmoreland Street, London W1M 8BA. 